
Arthritis During Elective Hand Surgery

Kevin M. Klifto, PharmD; Brian H. Cho, MD; Scott D. Lifchez MD

Department of Plastic and Reconstructive Surgery, The Johns Hopkins University School of Medicine, Baltimore, Maryland, USA, 21224

$\mathrm{PCOM}_{\odot}$

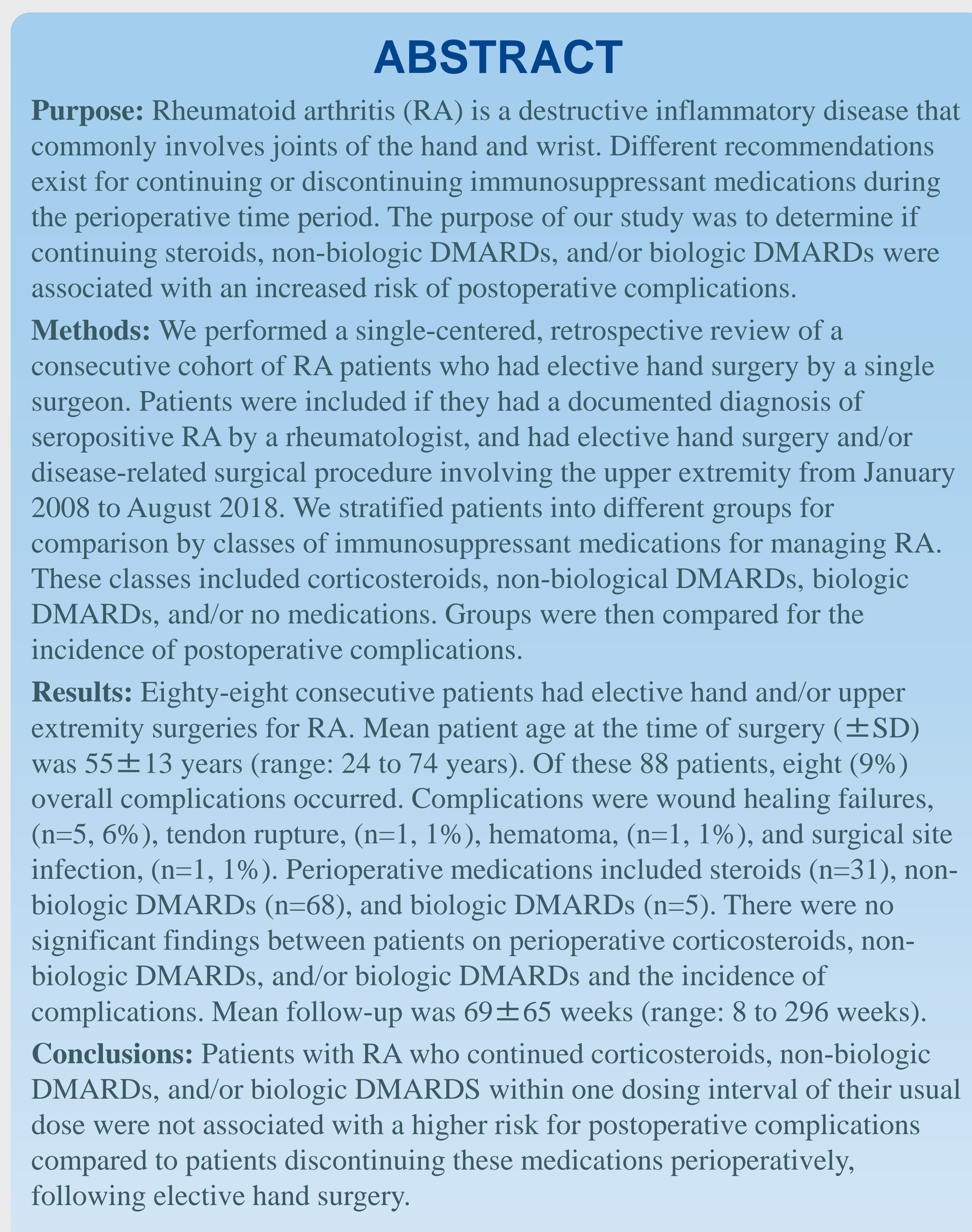

INTRODUCTION

Rheumatoid arthritis (RA) is the most common chronic destructive inflammatory disease. Synovial surfaces of the hand and wrist joints are destruction and pain, disability, systemic complications, poor quality of life, and a decreased lifespan. ${ }^{1}$ First-line medication management with
immunosuppressing disease-modifying antirheumatic drugs (DMARDs) has proven to improve survival and decrease surgical interventions in patients wit RA.
treat already damaged joins.

As the disease progresses, surgery may be required to decrease pain, correct deformities, and increase range of motion. ${ }^{2,4}$ Prior to any surgical procedure,
many surgeons discontinue immunosuppressant medications to prevent the many surgeons discontinue immunosuppressant medications to prevent the
complications associated with wound healing and surgical wound infections. ${ }^{10}$ Corticosteroids, non-biologic and biologic DMARDs are all classes of medications with different pharmacologic mechanisms and varying
immunosuppressant activity commonly taken by patients diagnosed with rheumatoid arthritis to prevent disease progression. ${ }^{3}$ Continuing steroids and
non-biologic DMARDs but holding biologic DMARD weeks ping hip or knee surgery is , but hip or knee surgery is currently recommended by the American College of
Rheumatology and American Association of Hip and Knee Surgeons and the
literature $1.3,11-14$ These recommendations include discontinuing etanerept literature. $1.3,11-14$ These recommendations include discontinuing etanerept 1week before surgery, infliximab, golimumab, tocilizumab, abatacept,
adalimumab, certolizumab, 4 weeks before surgery, and rituximab 8 weeks adalimumab, certo
before surgery. ${ }^{1,}, 1-14$

We could find no study in the literature that evaluated different combinations of perioperative immunosuppressant medications in a cohort of rheumatoid study was to determine if continuing steroids, non-biologic DMARDS, and/or complications. A secondary aim was to see what patient demographic specific complications in our patient population.

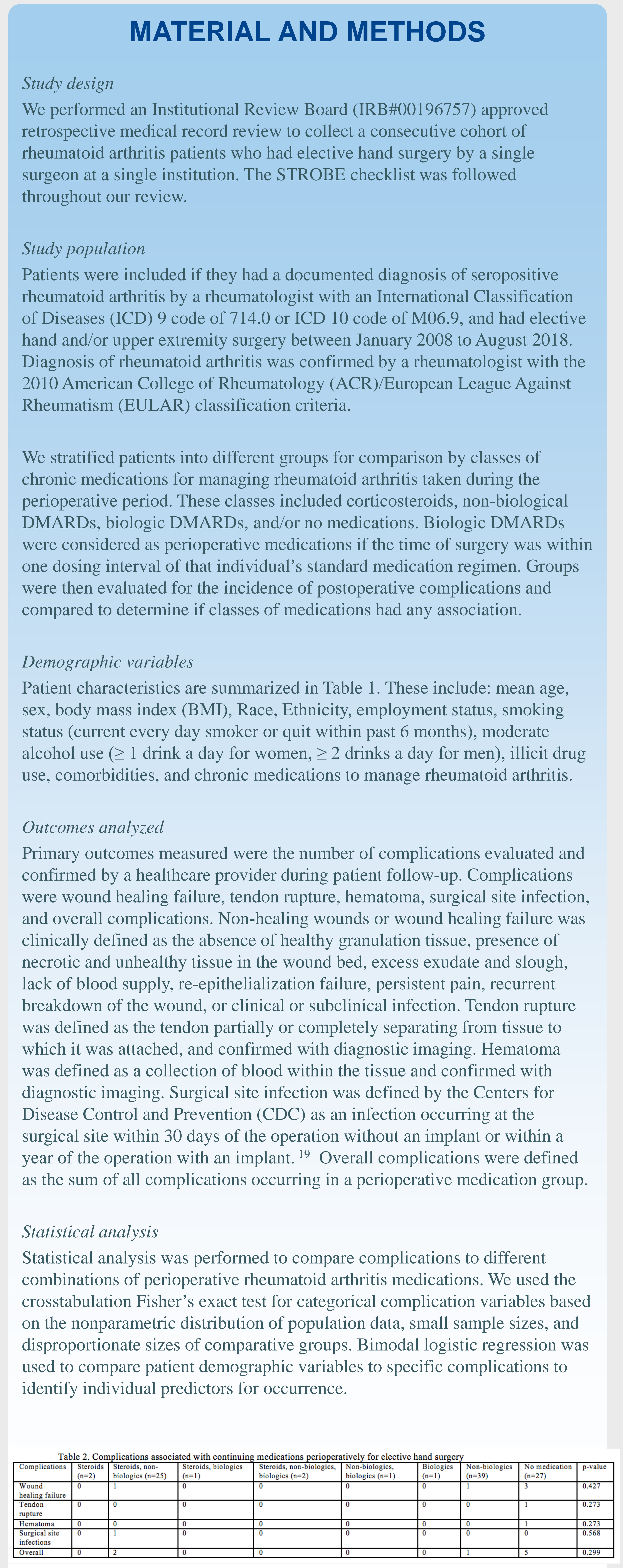

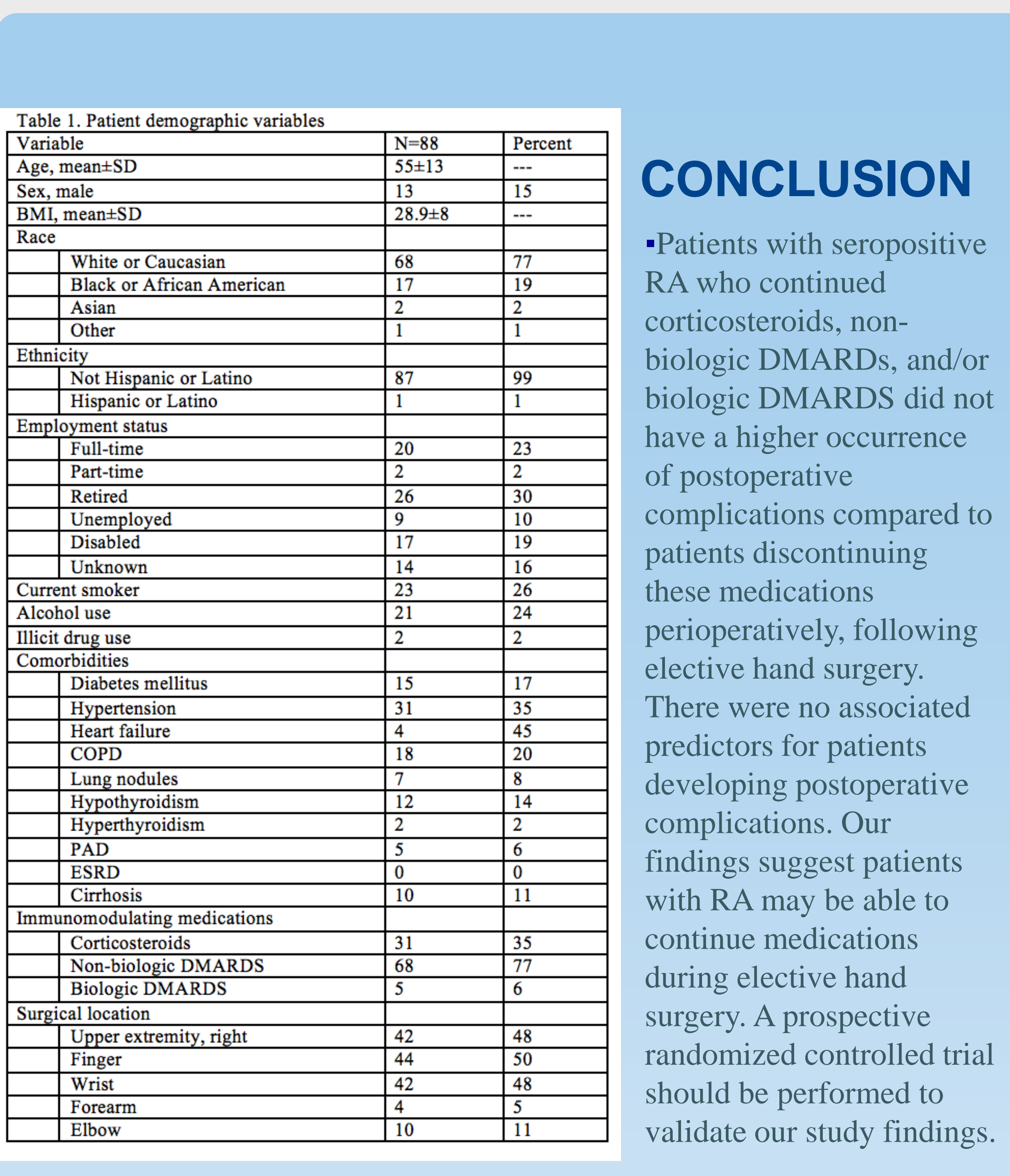

REFERENCES

One of the eight (13\%) complications was tendon rupture (Table 2). The extensor compartments, resection of left distal $y$ una and suspension of the ulnar shaft using a distally based extensor carpi ulnaris tendon. Three months following the procedure, magnetic resonance imaging (MRI) confirmed
rupture of the left extensor tendons of fingers 3,4 , and 5 . This one case rupture of the left extensor tendons of fingers 3,4 , and 5 . This one case
occurred in a patient taking no medications. The patient stated the ruptured occurred two months prior from no known inciting event. There were no significant increased risks of tendon rupture in patients taking no medicatio steroids, non-biologic DMARDs, and/or biologic DMARDs during the
perioperative period $(\mathrm{p}=\mathrm{NS})$. Following bimodal logistic regression ther

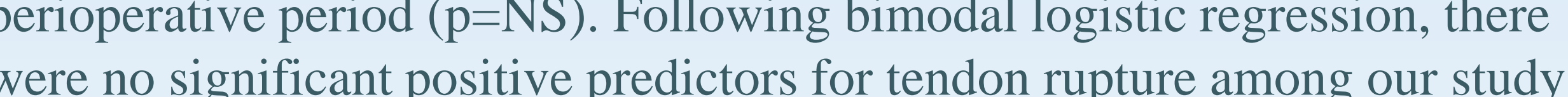
demographic variables $(\mathrm{p}=\mathrm{NS}$ )

Hematoma

One of the eight (13\%) complications was a hematoma (Table 2). This one case occurred in a patient taking no medications. There were no significant
increased risks of hematoma in patients taking no medications, steroids, nonbiologic DMARDs, and/or biologic DMARDs during the perioperative períd positive predictors for hematoma among our study demographic variables

\section{Surgical site infection}

One of the eight (13\%) complications was a surgical site infection (Table 2). There were no significicant increased risks of surgical site infections in patients perioperative period. Following bimodal logistic regression, there were no perioperative pertod. Following bimodal logistic regression, there were demographic variables $(\mathrm{p}=\mathrm{NS}$ )

Overall complications

There were eight overall complications in $88(9 \%)$ hand surgery cases (Table 2). Two (25\%) in patients taking steroids and non-biologics, one ( $25 \%$ ) in
patients taking only steroids, and five ( $63 \%$ ) in patients taking no medications patients taking only steroids, and five (63\%) in patients taking no medication
$(\mathrm{p}=\mathrm{NS})$. There were no significant increased risks of overall complications in patients taking steroids, non-biologic DMARDs, and/or biologic DMARDs were no significant positive predictors for overall complications among our study demographic variables ( $\mathrm{p}=\mathrm{NS}$ ) 